produced by a given bombardment is of the order $3 \times 10^{-21} \mathrm{~cm} .^{2}$. The cross-section calculated on the assumption that displacements are produced by direct collision with atomic nuclei lies between $5 \times 10^{-23}$ and $10^{-22} \mathrm{~cm} .{ }^{2}$. The measured cross-section gives a lower limit to the value pertaining to the principal displacement mechanism because there is not necessarily a one-one correspondence between the number of displacements and the number of colour centres produced. Dr. Varley used the relation between the cross-section $\sigma_{p}$ for a $p$-fold ionization of a given atom or ion and the cross-section $\sigma_{1}$ for a single ionization in the approximate formula

$$
\sigma_{p} \sim \sigma_{1}\left(\frac{\sigma_{1}}{A}\right)^{p-1}
$$

where $A$ is the 'area' of the atom. From the rangeenergy relation obtained experimentally, it appears that $\sigma_{1}$ is of the order $10^{-17} \mathrm{~cm}^{2} ; A$ can be taken as $\sim 10^{-15} \mathrm{~cm}^{2}$. By the substitution

$$
\sigma_{p} \sim 10^{-17}\left(10^{-2}\right)^{p-1}
$$

since $\sigma_{p} \sim 10^{-21} \mathrm{~cm}^{2}$, the approximation gives $p=3$ as the number of electrons stripped from one ion. It is thus indicated that the removal of three electrons from a given negative ion is a sufficiently probable process to account for the number of colour centres and, consequently, for displacements produced, if it be assumed that each one of these three. fold ionized ions is ejected from its lattice site and trapped at some other site in the crystal. The endstate can thus be halogen atoms in interstitial positions, trapped in positive-ion vacancies or at dislocations, and electrons trapped in the sites vacated by the halogen ions, these electrons giving rise to the colour centres. Dr. Varley concluded by remarking that vacancy pairs can lead to fast bleaching by acting as scavengers, but there is still much to be elucidated.

J. F. HINSLEY

${ }^{1}$ Varley, J. H. O., J. Nuclear Energy, 1, 130 (1954).

${ }^{2}$ Varley, J. H. O., Nature, 174, 886 (1954).

\title{
THE NATIONAL GEOGRAPHIC SOCIETY_PALOMAR OBSERVATORY SKY ATLAS
}

T HE first section of the Palomar Sky Atlas has recently been distributed to subscribers. This Atlas is being prepared from a survey of the sky north of declination $-27^{\circ}$, made with the 48 -inch Schmidt telesceis on Mount Palomar. This area of the sky has been photographed on 879 pairs of plates ; each plate of size 14 in. $\times 14$ in. covers an area of $7 \times 7$ degrees. The plate centres are separated by $6^{\circ}$, so that adjacent plates overlap by $\frac{1}{2}$ degree.

Each field is photographed in two colours in immediate succession. The blue exposures are obtained on the Eastman 103a0 emulsion, with a wave-length range of from $3500 \mathrm{~A}$. (the short-wave transmission limit of the correcting plate) to $5000 \mathrm{~A}$., the maximum sensitivity being at about $4100 \mathrm{~A}$. The red exposures are obtained on the Eastman $103 a E$ emulsion, combined with a red 'Plexiglass' filter, with a wave-length range of from $6200 \mathrm{~A}$. (the short-wave cut-off limit of the filter) to $6700 \mathrm{~A}$., the maximum sensitivity being at about $6500 \mathrm{~A}$.

The exposure times for each series were chosen to reach the faintest stars which can be recorded by the telescope under average observing conditions. These times were separately determined for each shipment of plates and range from 10 to $15 \mathrm{~min}$. for the blue exposures and from 40 to $60 \mathrm{~min}$. for the red. The photographic magnitude limit is $21 \cdot 1$ for the blue plates and 20.0 for the red. The magnitudes of stellar images on the blue plates are approximately on the same system as that of the international photographic magnitudes, and those of the images on the red plates are approximately on the same system as the red magnitudes of Kron and Smith. The differences between blue and red magnitudes of stars on the survey plates are approximately $\mathbf{1 . 6}$ times their international colour indices. Stars having an international colour index of 0.7 appear about equally bright on the blue and red plates. The scale of the plates is $67 \cdot 1$ seconds of are per millimetre.

Each pair of photographs of a field was inspected for quality before being accepted for reproduction in the atlas. If one member of a pair was rejected, the area was re-photographed in both colours. Many fields had to be photographed several times before both plates were passed as acceptable.

The photographs were first reproduced as positive transparencies. The photographic prints are in the form of negative reproductions, because they alone permit of satisfactory preservation of the plate limit. The prints were made by the Graphic Arts Facilities of the California Institute of Technology. Care has been taken to ensure that the faintest details at the limits of the plates are reproduced even at the price of inadequate reproduction of bright objects, so that the images of faint stars, galaxies and nebulosities, which are beyond the reach of smaller instruments, are preserved.

After extensive tests and trials, Eastman Kodak Finegrain Positive glass plates were selected for the positive transparencies and Eastman Kodak Unicontrast double-weight paper for the negative prints. The contrast of the paper is gamma $2 \cdot 0$, practically independent of development. The original plates show considerable variation in background density, which depends upon the sky brightness. Original plates with background densities less than $0 \cdot 3$ and greater than 0.9 were found to give inferior results and were rejected. The most satisfactory reproduction was found to be obtained by printing all positives to a density of $1 \cdot 4$. The paper prints were found to be most satisfactory with a density of 0.2 or slightly higher.

On the back of each print is stamped the nominal position of the plate centre for epoch 1855. In the north-east corner of each photograph is a label giving the serial number of the plate, the date of the exposure and the 1950 co-ordinates of the field centre.

The survey was made financially possible by generous grants from the National Geographic Society, which provided the photographic materials and equipment, the salaries of the personnel employed on the survey, and the cost of producing two contact positives on glass of each survey photograph. The 
observing time with the 48-inch Schmidt telescope required to obtain the survey photographs was made available by the Palomar Observatory of the California Institute of Technology. Approximately eight years of observing time (1949-56) will be required to obtain the negatives. The copyright for the Atlas is held by the National Geographic Society.

The Atlas has been made available at a price that covers only the cost of production and handling of the prints. The estimated cost was 2,000 dollars per set of 1,758 prints ( 879 blue exposures and 879 red exposures) if fifty copies were ordered and 1,600 dollars per set if a hundred copies were ordered. The actual price can only be established after the printing is completed. Cancellation of orders will be accepted if the estimated price is higher than 2,000 dollars. Only one printing of the Atlas is planned, and no orders could be accepted after October 1, 1954, when the reproduction was commenced.

The Atlas will provide an invaluable working tool for observatories that possess copies. The Franklin Adams Charts, which covered the whole sky but which were made from originals on blue-sensitive emulsions only and which did not reach nearly to such a faint magnitude as the Palomar survey, have for many years proved of great value for many purposes. The photographs in two colours of the present survey and the faint-magnitude limit reached are of special importance : it is readily possible, for example, to recognize red stars and blue stars by the comparison of the sizes of their images on the blue and red photographs, while for such studies as those of interstellar absorption and the distribution of faint galaxies the reproductions will prove most useful.

It is recommended that, in order to prevent damaging the prints when in use, they should be covered with glass plates. The most satisfactory illumination is with the light source low and to the rear.

In making this Atlas available and at a reasonable price, astronomy will owe a great debt to the National Geographic Society and to the Mount Palomar Observatory.

H. SPENCER JONES

\section{OBITUARIES}

\section{Prof. A. R. Radcliffe-Brown}

With the death on October 24 at the age of seventy-four of Prof. Alfred Reginald RadcliffeBrown, emeritus professor in the University of Oxford, comparative social anthropology has lost its leader and foremost exponent. An intellectual heir of Montesquieu, Comte and Spencer, he represented the theoretical tradition which culminated in Durkheim, flourished with the French sociological school and was submitted to the test of empirical fieldobservations by British anthropology.

Radcliffe-Brown read mental and moral science as a scholar of Trinity College, Cambridge, and he worked with both Rivers and Haddon. At the age of twenty-six, after his election to the Antony Wilkin studentship in ethnology, he carried out research among the Andaman Islanders. His book under that title, completed in 1913 and published in 1922, is a brilliant analysis of ritual and of social organization. Together with the account of the Cambridge expedition to the Torres Straits and with Malinowski's "Argonauts of the Western Pacific", it inaugurated a new departure and pointed the way to the intensive field-work characteristic of modern anthropology. Apart from a number of important articles, this pioneering work marked Radcliffe-Brown's principal contribution to the field-study of primitive peoples. This was due mainly to his conception of social anthropology as a natural science of society and, within this discipline, to his personal attraction to inquiries aiming at the establishment of general principles.

Radcliffe-Brown propagated his views with zeal, and this left him with little time for field-investigations. $\mathrm{He}$ was appointed the first professor of social anthropology in the University of Cape Town (1921), head of the newly created Department at Sydney (1926), where he founded and edited Oceania, and professor at Chicago in 1931. Between 1937 and 1946 he occupied the newly created chair at Oxford, where he was a Fellow of All Souls, which had taken a leading part in its establishment. $\mathrm{He}$ also taught social anthropology at Cambridge, London, Birmingham, Pretoria, Johannesburg, Yenching, São Paulo, Alexandria, Manchester and Grahamstown.

A man of distinctive appearance, he combined a natural charm of manner, kindness to his colleagues and generosity to his juniors with an innate reserve which, at times, bordered on aloofness. He was happiest and most relaxed in the social climate of a 'salon', where he could discuss with equal verve an exotic recipe or his personal conception uf Confucian philosophy.

Radcliffe-Brown was by temperament, method and necessity a thinker rather than an observer, a conversationalist, lecturer and essayist rather than the writer of lengthy treatises. He wrote with litotes and crystalline clarity, and his essays on kinship, taboo, primitive law and social structure have proved invaluable in helping to lay the anthropological foundations of the subject; and they are indispens. able in its present-day teaching.

$$
\text { J. G. Peristiany }
$$

\section{Dr. G. Placzek}

Dr. George Praczex, of the Institute of Advanced Study, Princeton, who died at Zurich on October 16 , was born in 1905 in Brunn (now Brno, Czechoslovakia). After studying theoretical physics in Vienna and Prague, he moved around Europe, working in Utrecht, Leipzig, Copenhagen and Paris, among other places. He spent a year at the Hebrew University, Jerusalem. In 1938 he went to Cornell University, and later worked on atomic energy at Cambridge, Montreal and Los Alamos. After two years in the research laboratory of the General Electric Company at Schenectady, he joined the Institute for Advanced Study in Princeton in 1948. $\mathrm{He}$ married in 1943.

Placzek's earliest major work was on the theory of the Raman effect, on which his review article became a classic. This led to a study of molecular rotational states, then a fruitful tool for determining nuclear spins. Later he turned to neutron problems. With $O$. R. Frisch he first proved that resonance-levels for neutron capture have extremely low energies. In collaboration with others, he helped to clarify the dispersion formula for nuclear reactions. Then came a study of the slowing-down of neutrons, which was started for its intrinsic interest, but proved of great importance for work on atomic energy. This led to study of the integral equations of neutron multiplica- 